\title{
Neurovascular Complications in COVID-19 Infection: Case Series
}

\author{
(D) A.M. Franceschi, (D) R. Arora, (D) R. Wilson, (D) G. Giliberto, (D) R.B. Libman, and (D) M. Castillo
}

\section{ABSTRACT}

SUMMARY: We present a series of 10 hospitalized patients with confirmed coronavirus 2019 infections who developed severe neurovascular complications and discuss the possible reasons for these findings and their relationship to the novel Severe Acute Respiratory Syndrome coronavirus 2 infection.

ABBREVIATIONS: ACE2 = angiotensin-converting enzyme 2; COVID-19 = coronavirus 2019; CRP = C-reactive protein; CSS = cytokine storm syndrome; $\mathrm{ICU}=$ intensive care unit; $\mathrm{LDH}$ = lactate dehydrogenase; PCR = polymerase chain reaction; SARS-CoV-2 = Severe Acute Respiratory Syndrome coronavirus 2

C oronavirus disease 2019 (COVID-19) is a predominantly acute respiratory disease caused by the novel Severe Acute Respiratory Syndrome coronavirus 2 (SARS-CoV-2). ${ }^{1}$ In addition to progressive atypical respiratory system distress, other organ systems including the CNS may be affected in part due to the marked affinity of the virus for the angiotensin-converting enzyme 2 (ACE2) receptors. ${ }^{2,3}$ Furthermore, a subgroup of patients with severe COVID-19 develop cytokine storm syndrome (CSS), characterized by hyperinflammation due to rapid accumulation of T-cells and macrophages, resulting in the release of massive levels of cytokines into the bloodstream to eliminate the offending pathogen, causing numerous clinical manifestations including atypical respiratory system distress and fever, progressing to widespread multiorgan dysfunction. ${ }^{4-7}$

An increasingly recognized feature of COVID-19 infection is a thromboembolic diathesis, often resulting in brain ischemia, even in young patients despite anticoagulation/antiplatelet treatment. The comorbidity of pneumonia and thromboembolic complications in patients with COVID-19 may be explained, in part, by a causal relationship of severe inflammation and infection as precipitating factors. ${ }^{6-8}$

Here, we present the neurovascular complications in 10 hospitalized patients with COVID-19 infection at our institutions.

Received May 4, 2020; accepted after revision May 22.

From the Neuroradiology Section (A.M.F.), Departments of Radiology and Neurology (R.A., L.G., R.B.L.), Donald and Barbara Zucker School of Medicine at Hofstra/Northwell Health, Manhasset, New York; and Neuroradiology Section (R.W., M.C.), Department of Radiology, The University of North Carolina School of Medicine, Chapel Hill, North Carolina.

Please address correspondence to Ana M. Franceschi, MD, Department of Radiology, 300 Community Dr, Manhasset, NY, 11030-3816; e-mail: afranceschi@northwell.edu

- Indicates open access to non-subscribers at www.ajnr.org

http://dx.doi.org/10.3174/ajnr.A6655
Available neuroimaging studies including head CT, head and neck CTA, and brain MR imaging were reviewed.

\section{Case Reports}

Patient 1. A 59-year-old woman with a medical history of hypertension and morbid obesity was admitted with hypoxic respiratory failure requiring mechanical ventilation.

Initially, she experienced cough but no fever and went to an emergency department and was advised to get a head CT but left against medical advice. She drove to a different state and developed weakness and difficulty moving her legs, increased thirst, and dyspnea. She presented to our emergency department with acute hypoxic respiratory failure, fever, acute renal failure, and bilateral lung opacities on chest $\mathrm{x}$-ray. She tested positive for COVID-19 by polymerase chain reaction (PCR) and was subsequently intubated and transferred to the intensive care unit (ICU), where she developed septic shock and labile blood pressures. Laboratory values included elevated D-dimer, lactate dehydrogenase (LDH), C-reactive protein (CRP), and ferritin levels, suggesting CSS. Chest CT demonstrated diffusely scattered ground-glass opacities in the nondependent lung with consolidation in the dependent lung parenchyma compatible with atypical respiratory system distress in the setting of COVID-19 infection. She developed a sudden right hemineglect, and head CT demonstrated focal low attenuation in the left posterior frontal/anterior parietal lobes consistent with an infarction (Fig 1). The patient did not improve and died 21 days following admission.

Patient 2. A 37-year-old man with a history of hypertension and morbid obesity presented to an outside hospital with fever and difficulty breathing, initially attributed to influenza. PCR testing 
was performed and was positive for COVID-19, and he was admitted for medical care.

He developed acute renal failure and required mechanical ventilation with increasingly difficult oxygenation. Laboratory findings were supportive of CSS with elevated D-dimer, LDH, $\mathrm{CRP}$, and ferritin levels. Chest CT revealed diffusely scattered consolidative and ground-glass opacities in the nondependent lung and consolidations in the dependent lung consistent with atypical infection and atypical respiratory system distress. After weaning from sedatives and paralytics, he was unresponsive for several hours and again was transferred to the ICU where he was noted to have no movement of the extremities and dilated, fixed pupils. Noncontrast CT of the head showed intra-axial hemorrhages in the cerebellar hemispheres and diffuse cerebral edema (Fig 2). A neurologist confirmed brain death.

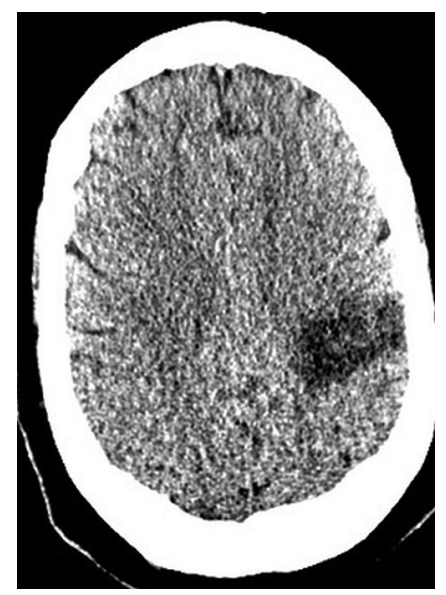

FIG 1. Axial noncontrast head CT demonstrates focal low attenuation in the left posterior frontal/anterior parietal lobes in the region consistent with an acute infarction.

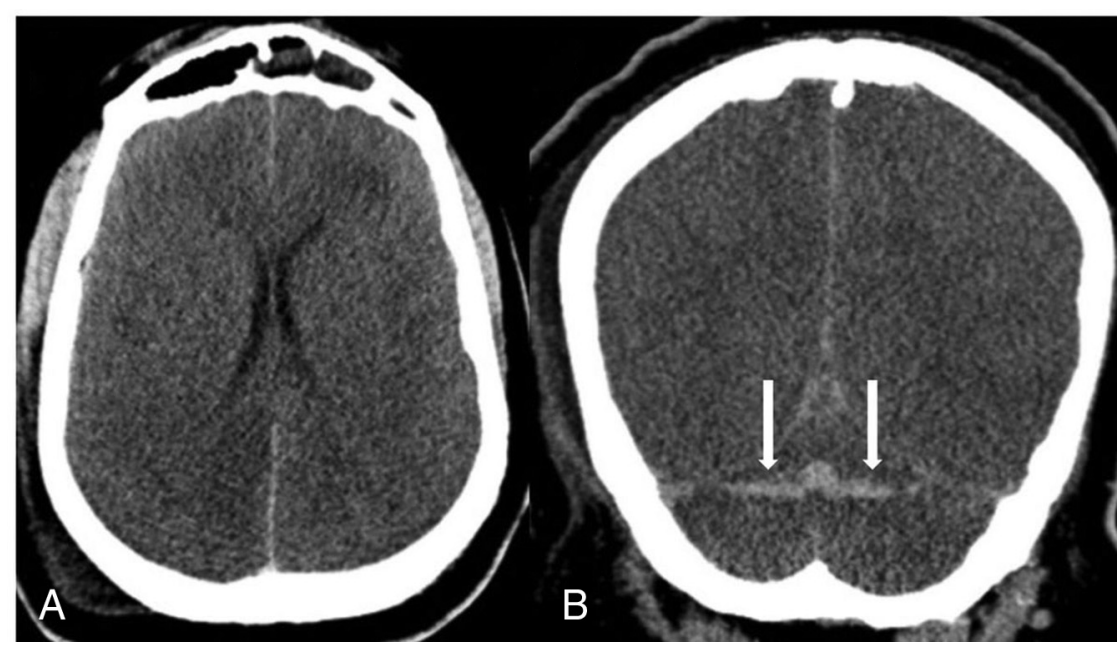

FIG 2. Axial $(A)$ and coronal $(B)$ noncontrast $C T$ demonstrates diffuse cerebral edema. There are horizontally oriented intra-axial hemorrhages (arrows) in the cerebellar hemispheres on the coronal view.
Patient 3. A 33-year-old man with no relevant medical history presented following 12 days of self-treatment with acetaminophen for respiratory symptoms. On the day of admission, he developed sudden-onset left-sided weakness and numbness. PCR testing was positive for COVID-19, and he was admitted.

Chest CT revealed diffuse consolidative and ground-glass opacities bilaterally. Laboratory values were concerning for sepsis (lactate, $9.9 \mathrm{~mm} / \mathrm{L}$; CRP, $312 \mathrm{mg} / \mathrm{L}$ ) and coagulopathy (D-dimer, >50,000 ng/mL; ferritin, $1636 \mathrm{ng} / \mathrm{mL}$ ). He developed septic shock requiring vasopressor support. He was found to be in severe acute hypoxic respiratory failure $\left(\mathrm{O}_{2}\right.$ saturation, $11 \%$ ), was intubated, and was noted to have a right forced-gaze deviation and left hemiparesis. He was treated with IV tPA for suspected acute right MCA infarction and placed on extracorporeal membrane oxygenation. Head CT demonstrated subacute infarctions in the right frontal, right parietal, and right parieto-occipital regions with edema and mass effect resulting in subfalcine herniation and an associated small hemorrhage in the right parietal region (Fig 3). CTA of the head and neck demonstrated no major vessel narrowing or occlusion. The patient remains in the neurologic ICU while on argatroban (direct thrombin inhibitor) drip and a $3 \%$ hypertonic saline drip for cerebral edema.

Patient 4. A 71-year-old man with diabetes mellitus type 2, hyperlipidemia, and hypertension presented with altered mental status and 2 days of fever and cough. Due to exposure to COVID19 from a family member, he was tested and was positive for the disease.

Laboratory results showed hypoglycemia and acute renal failure. Noncontrast head CT and CTA findings were unremarkable. MR imaging of the brain showed no acute findings. He remained stable the first week and underwent a course of hydroxychloroquine and an interleukin-2 receptor antagonist with improvement in his respiratory status. On hospital day 8 , he developed acute encephalopathy and respiratory failure and was intubated. His D-dimer level spiked to $31,000 \mathrm{ng} / \mathrm{mL}$, and chest $\mathrm{x}$-ray showed bilateral multifocal pneumonia and pulmonary edema. Noncontrast head CT was performed and demonstrated a large left MCA infarction with evolving low attenuation in the left frontal, parietal, and temporal lobes extending to the left basal ganglia (Fig 4). He subsequently developed multiorgan failure and died.

Patient 5. A 40-year-old man with recently diagnosed diabetes mellitus, hypertension, and hyperlipidemia with a PCR positive for COVID-19 was discharged on supportive treatment after a brief hospital stay for a cough. 


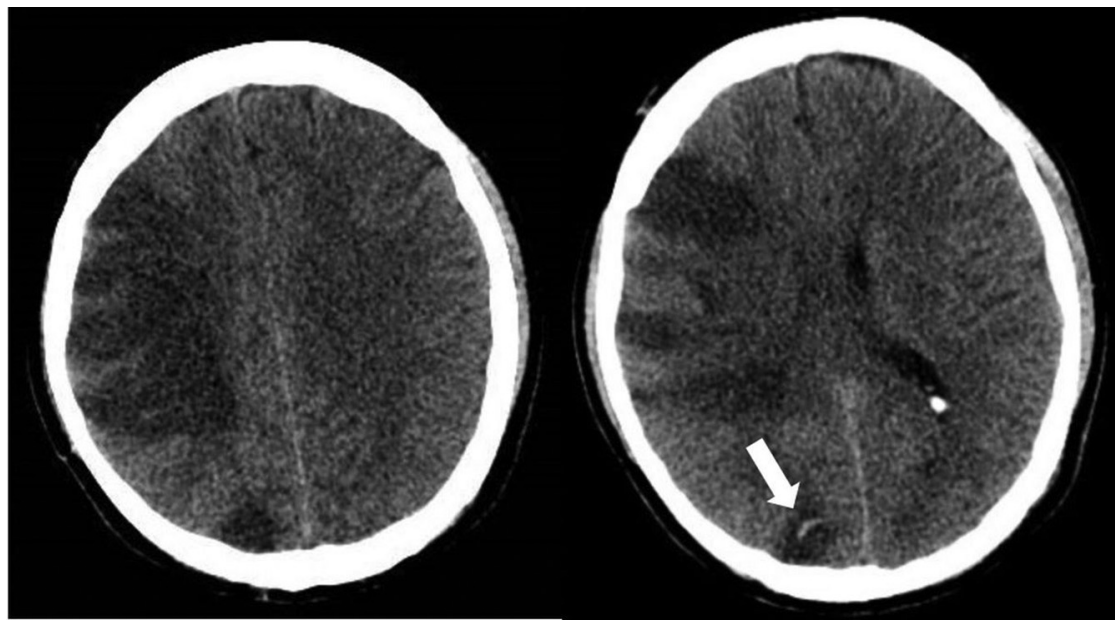

FIG 3. Axial noncontrast $C T$ demonstrates acute infarctions in the right frontal and parietal regions. There is a subcentimeter hemorrhage (arrow) in the right parietal region.

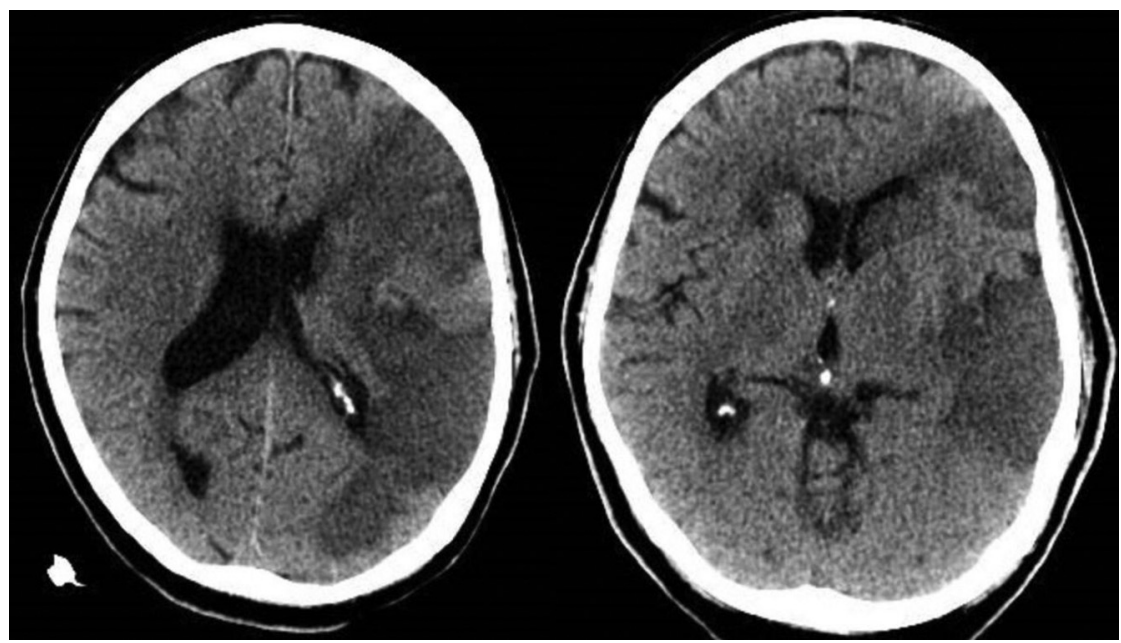

FIG 4. Axial noncontrast $C T$ demonstrates acute infarctions in the left frontal, parietal, and temporal lobes extending to the left basal ganglia and insular cortex, with additional small infarcts in the right anterior limb of the internal capsule and right frontal subcortical white matter.
Thereafter, he developed altered mental status, bilateral loss of vision, and headache. Laboratory results were notable for lymphocytosis and $1 \%$ bands, hemoglobin and platelets elevated at $17.6 \mathrm{~g} / \mathrm{dL}$ and 762,000 respectively, international normalized ratio elevated at 1.39, and fibrinogen level elevated at 902 . His D-dimer level was elevated at $3115 \mathrm{ng} / \mathrm{mL}$, with the following values: lactate, $7.5 \mathrm{~mm} / \mathrm{L}$; blood urea nitrogen/creatinine, $13 \mathrm{mg} / \mathrm{dL} /$ $1.37 \mathrm{mg} / \mathrm{dL}$ (baseline creatinine, 0.8 $\mathrm{mg} / \mathrm{dL}$ ); aspartate transaminase, slightly elevated at $56 \mathrm{u} / \mathrm{L}$; alanine aminotransferase, normal at $67 \mathrm{u} / \mathrm{L}$; and $\mathrm{LDH}$, elevated at $577 \mathrm{u} / \mathrm{L}$. He developed a presumed CSS and was intubated and moved to the ICU. Noncontrast head CT showed low attenuation in the parietal and temporal lobes compatible with acute infarcts (Fig 5A). A head CT venogram showed occlusion of the right internal carotid artery extending to the supraclinoid segment where there was reconstitution of flow through collaterals (Fig 5B). CTA of the neck demonstrated filling defects in the distal common carotid arteries extending to the bulbs and external carotid arteries (Fig 5C). There was occlusion of the right internal carotid artery at the C2 level. CT venogram findings were normal.

The patient remains in critical condition in the ICU on full-dose anticoagulation without improvement in his neurologic status.

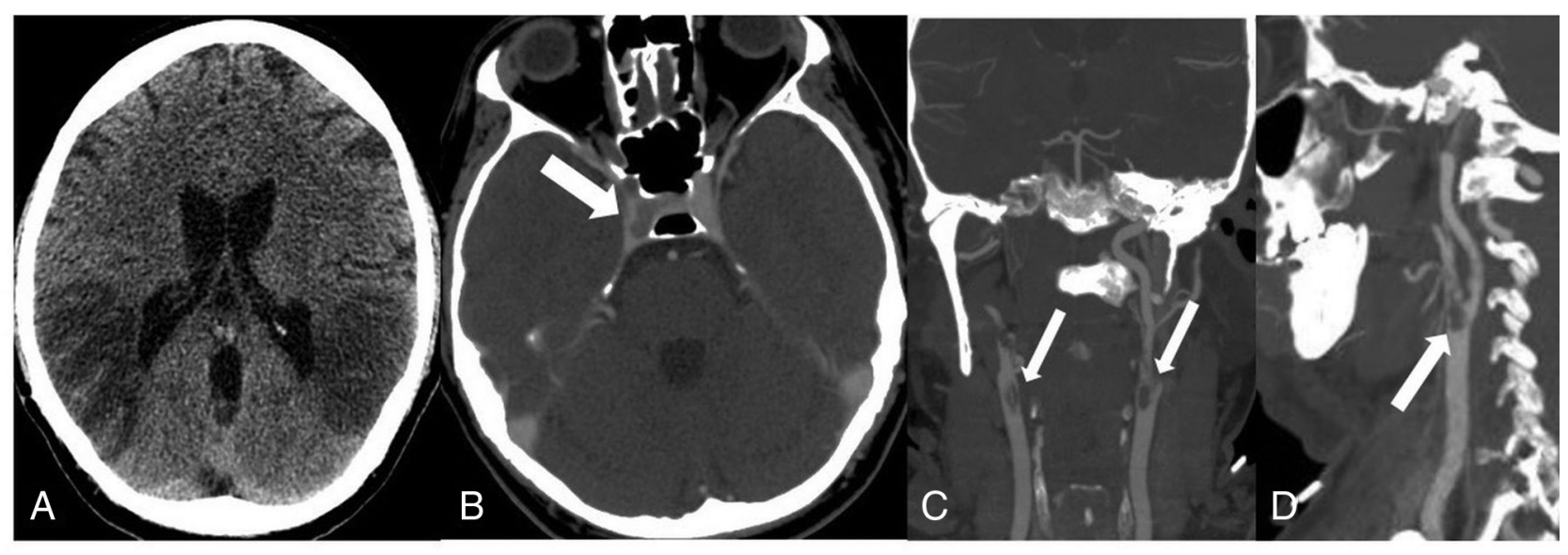

FIG 5. Noncontrast CT $(A)$ demonstrates low attenuation in the parietal and temporal lobes compatible with acute infarctions. Head CTV (B) demonstrates occlusion of the cavernous segment of the right internal carotid artery (arrow). Neck CTA ( $C$ and $D)$ shows extensive intraluminal thrombi (arrows) in the distal common carotid arteries, extending to the carotid bulbs and external carotid arteries. 


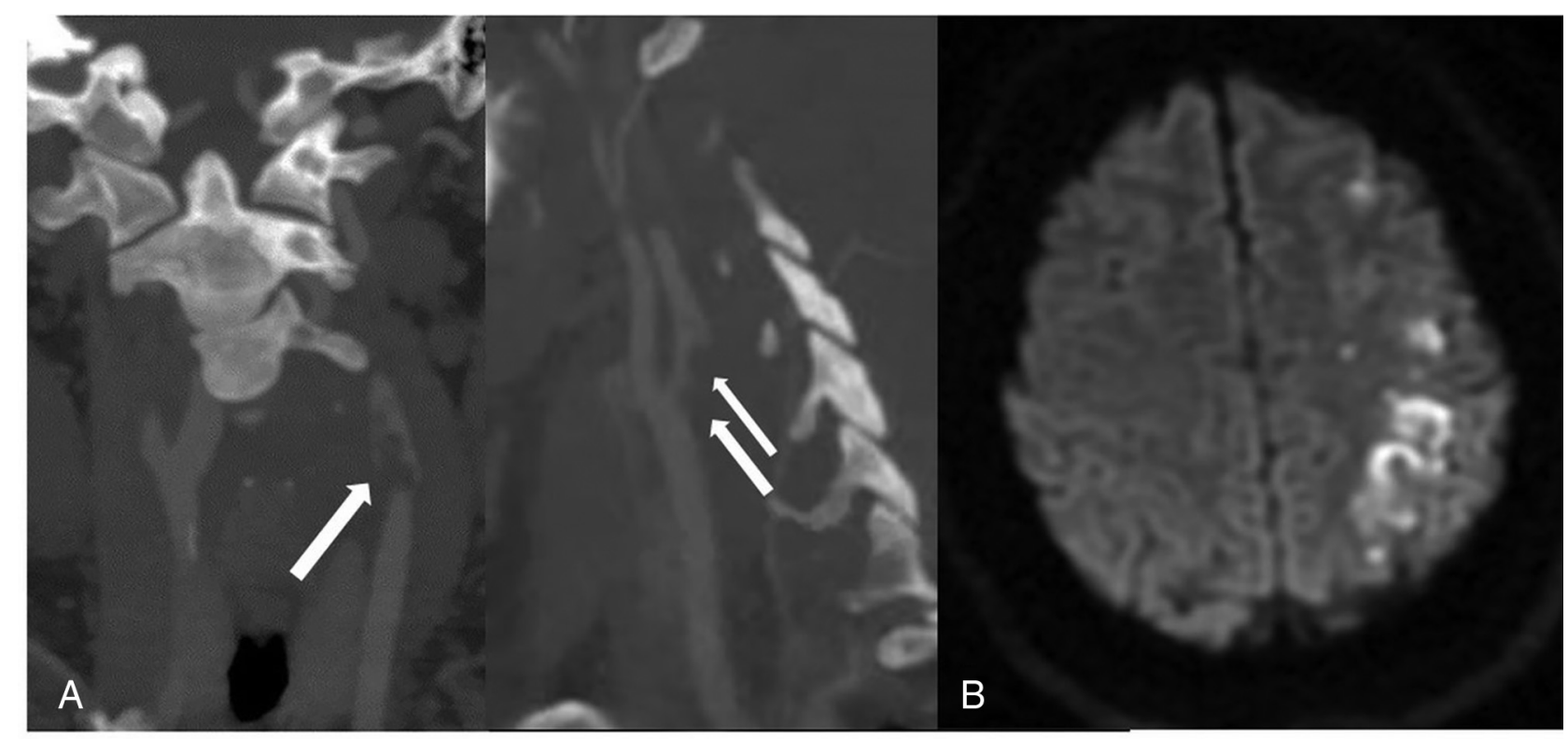

FIG 6. Coronal and sagittal neck CTA (A) demonstrates nonocclusive intraluminal thrombus (arrows) extending from the left common carotid artery to the proximal internal carotid artery. Axial DWI $(B)$ demonstrates acute infarcts in the left frontal and parietal regions.

Patient 6. A 37-year-old woman with a medical history of morbid obesity presented with right upper extremity weakness, slurred speech, and headaches. She reported chest congestion for 1 week prior, and a subsequently obtained PCR was positive for COVID19. She was admitted for hypoxic respiratory failure, but mechanical ventilation was not required. Laboratory values including Ddimer levels were within normal limits. Hypercoagulable workup revealed a weakly positive immunoglobulin $\mathrm{M}$ anticardiolipin antibody with negative findings on a dilute Russell viper venom time screen.

Noncontrast head CT and neck CTA showed intraluminal thrombus extending from the left common carotid artery into the proximal internal carotid artery (Fig 6A). Brain MR imaging revealed areas of restricted diffusion in the left frontal and parieto-occipital regions consistent with acute infarctions (Fig 6B). She remained hemodynamically stable throughout her hospital stay and was discharged on full-dose anticoagulation.

Patient 7. A 71-year-old man with a medical history of asthma, hypertension, and atrial fibrillation presented with fever, shortness of breath, and dry cough for 10 days, followed by a syncopal episode. PCR for COVID-19 was positive.

Chest CT revealed bilateral linear and ground-glass opacities with a peripheral predominance. Abnormal laboratory values included CRP, $25 \mathrm{mg} / \mathrm{L}$; LDH, $474 \mathrm{~mm} / \mathrm{L}$; and serum ferritin, 602 $\mathrm{ng} / \mathrm{mL}$. He developed acute respiratory failure with hypoxia despite $\mathrm{O}_{2}$ supplementation and was intubated. He was enrolled in an anti-interleukin 6 antibody treatment study and also received hydroxychloroquine and a course of methylprednisolone, resulting in improvement in his respiratory status. He was extubated, after which he was noted to be encephalopathic. Head CT revealed focal low attenuation in the right centrum semiovale and right frontal subcortical region, suggestive of infarctions
(Fig 7A, -B). Brain MR imaging demonstrated acute and subacute infarcts in the frontal lobes (right greater than left), bilateral centrum semiovale, right corona radiata, occipital lobes, and left cerebellar hemisphere (Fig 7C-7E). Concurrently performed MRA of the head and neck demonstrated no major vessel narrowing or occlusion. He remains in the ICU and is able to follow simple commands.

Patient 8. A 62-year-old woman with morbid obesity, diabetes mellitus type 2, hypertension, and hyperlipidemia presented with cough and fever and an outside chest CT with bilateral ground-glass opacities in a posterior and peripheral distribution.

PCR for COVID-19 was twice negative. Laboratory values were significant for elevated CRP, ferritin, and LDH, suggestive of CSS. Her D-dimer level was 12,000 ng/mL. The patient's oxygenation deteriorated, and she was intubated.

Ten days later, she developed altered mental status, and a head CT demonstrated low attenuation in the left occipital lobe (Fig 8A). Neck and head CTA findings were unremarkable. MR imaging showed restricted diffusion in the left occipital and parietal lobes. Pial enhancement was noted overlying the left occipital infarct as well as diffuse dural enhancement. SWI showed numerous microhemorrhages in the cerebral hemispheres, left cerebellum, and left occipital lobe (Fig $8 B-8 E$ ). She ultimately tested PCR-positive for COVID-19. Her respiratory status improved, and she was extubated. She was maintained on full-dose anticoagulation with enoxaparin and was discharged to a rehabilitation facility.

Patient 9. A 73-year-old man with no significant medical history presented with fever and nonproductive cough for 1 week. Given the local high-prevalence region, a PCR for COVID-19 was performed and was positive. Admission chest x-ray demonstrated bilateral lung opacities. Abnormal laboratory results 


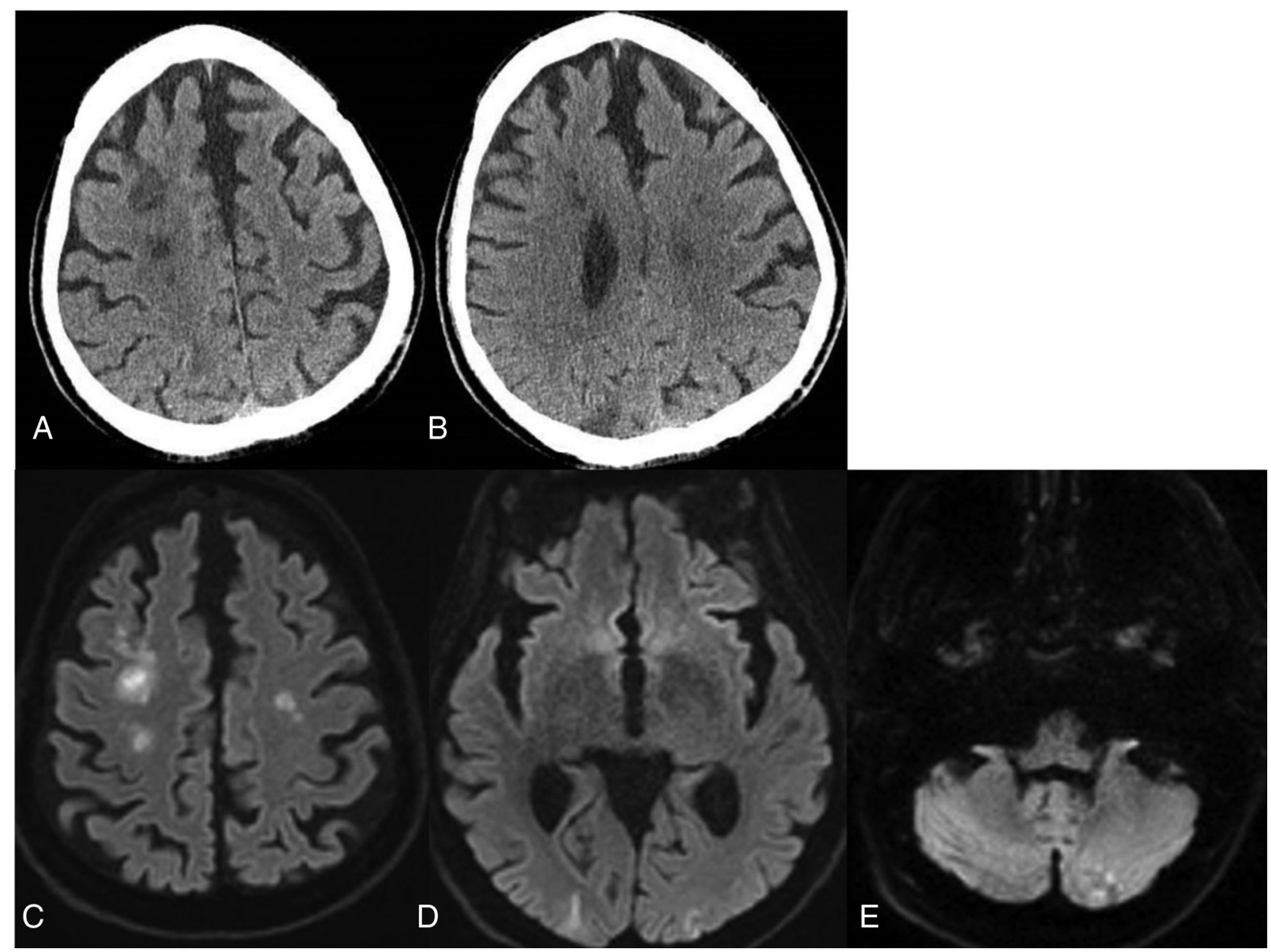

FIG 7. Noncontrast CT demonstrates focal low attenuation in the right frontal centrum semiovale and right frontal subcortical region $(A)$ and a wedge-shaped area of low attenuation in the right parietal region (B). DWI demonstrate small infarcts in the frontal lobes $(C)$, both occipital lobes $(D)$, and the left cerebellum $(E)$.

included the following values: elevated $\mathrm{D}$-dimer, $27,000 \mathrm{ng} / \mathrm{mL}$; CRP, $25 \mathrm{mg} / \mathrm{L}$; lactate, $3.1 \mathrm{~mm} /$; and pro-B-type natriuretic peptide, 9550 . He was intubated due to acute hypoxic respiratory failure and treated with an interleukin-2 receptor antagonist, steroids, and hydroxychloroquine.

Chest CT showed worsening pneumonia with diffuse, consolidative, and ground-glass opacities throughout both lungs. He then developed altered mental status and seizures, and a head CT showed focal low attenuation in the occipital and right frontal lobes, compatible with infarctions (Fig 9). CTA of the head and neck was not performed due to the patient's poor overall condition. During the next 2 days, his respiratory status deteriorated and he died.

Patient 10. A 62-year-old woman with a medical history of emphysema and lung cancer presented with acute encephalopathy and hypoxic respiratory failure requiring intubation and ventilator support. A PCR for COVID-19 was positive.

Abnormal laboratory results included mild leukocytosis (white blood cell count, $12 \times 10^{3} / \mathrm{mm}^{3}$ ); elevated D-dimer level, $5628 \mathrm{ng} / \mathrm{dL}$; elevated inflammatory markers (CRP and ferritin); elevated troponins; and marked respiratory acidosis.
A chest $\mathrm{x}$-ray showed bilateral opacities. Head CT demonstrated extensive low attenuation in the right frontal, temporal, and parietal regions consistent with infarction (Fig 10A). There was also subtle high attenuation in the right MCA (Fig 10B).

Head and neck CTA showed thrombus leading to occlusion of the right internal carotid artery. Intracranially, there was occlusion of the right internal carotid and middle cerebral arteries (Fig $10 C,-D)$. Follow-up head CT on the same day revealed a right middle cerebral artery territory acute infarction and areas of low attenuation in the left temporal region and cerebellar hemispheres (Fig 10E-10G).

Several days later, she developed profound refractory hypotension and severe hypoxia despite high-dose vasopressors and maximum ventilator support, and she died.

\section{DISCUSSION}

According to the World Health Organization, the ongoing COVID-19 global pandemic has infected $>3$ million people worldwide, resulting in nearly 218,000 deaths as of late April 2020 , with cases reported in 213 countries, areas, and territories. ${ }^{1}$ Various risk factors have been reported as predictors of 


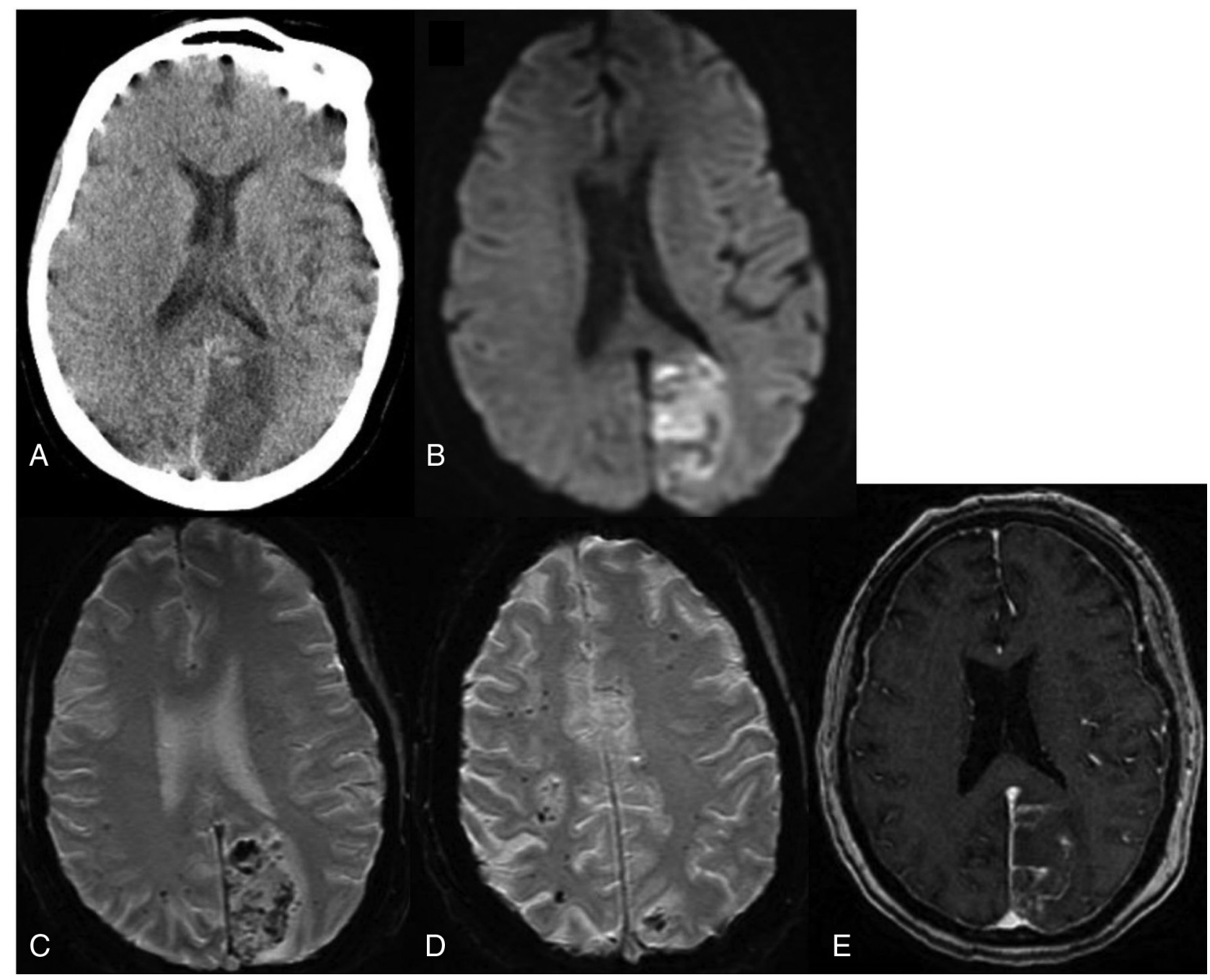

FIG 8. Noncontrast CT (A) demonstrates low attenuation in the left occipital lobe. DWI (B) confirms a left occipital infarction. Corresponding SWI (C) shows confluent hemorrhages in the region of the acute infarction. SWI through the centrum semiovale (D) shows numerous bilateral microhemorrhages. Postcontrast $\Pi$-weighted image $(E)$ shows leptomeningeal enhancement overlying the left occipital infarction and diffuse dural enhancement.

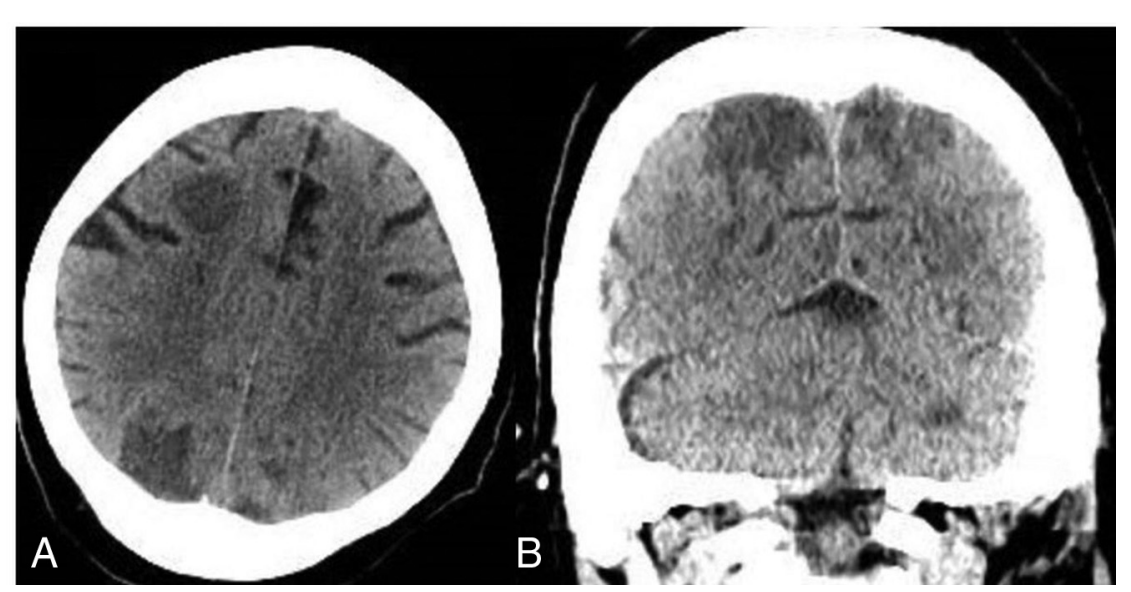

FIG 9. Non-contrast CT axial $(A)$ and coronal $(B)$ views demonstrate focal low attenuation in the parietal and right frontal lobes compatible with acute infarctions.

mortality and poor outcome, including advanced age, obesity, pre-existing cardiovascular or cerebrovascular diseases, and underlying immunosuppression., 9
Given the propensity of COVID19 infection to involve the respiratory system, initial scientific effort in diagnosis and therapeutics focused on the respiratory aspects of the disease. ${ }^{11}$ There is increasing evidence that thrombi are a major cause of multisystem organ dysfunction, including respiratory failure in severe cases of infection. According to several publications, clotting plays a major role in disease severity and mortality. ${ }^{12,13}$ In addition to arterial clots, others have observed high rates of venous thromboembolism in critically ill patients who otherwise lack the classic risk factors for venous thromboembolism. ${ }^{10}$

A postmortem examination of 1 patient with severe COVID-19 disease revealed numerous pulmonary microthrombi. One feature of these diffuse capillary thrombi was the presence of 


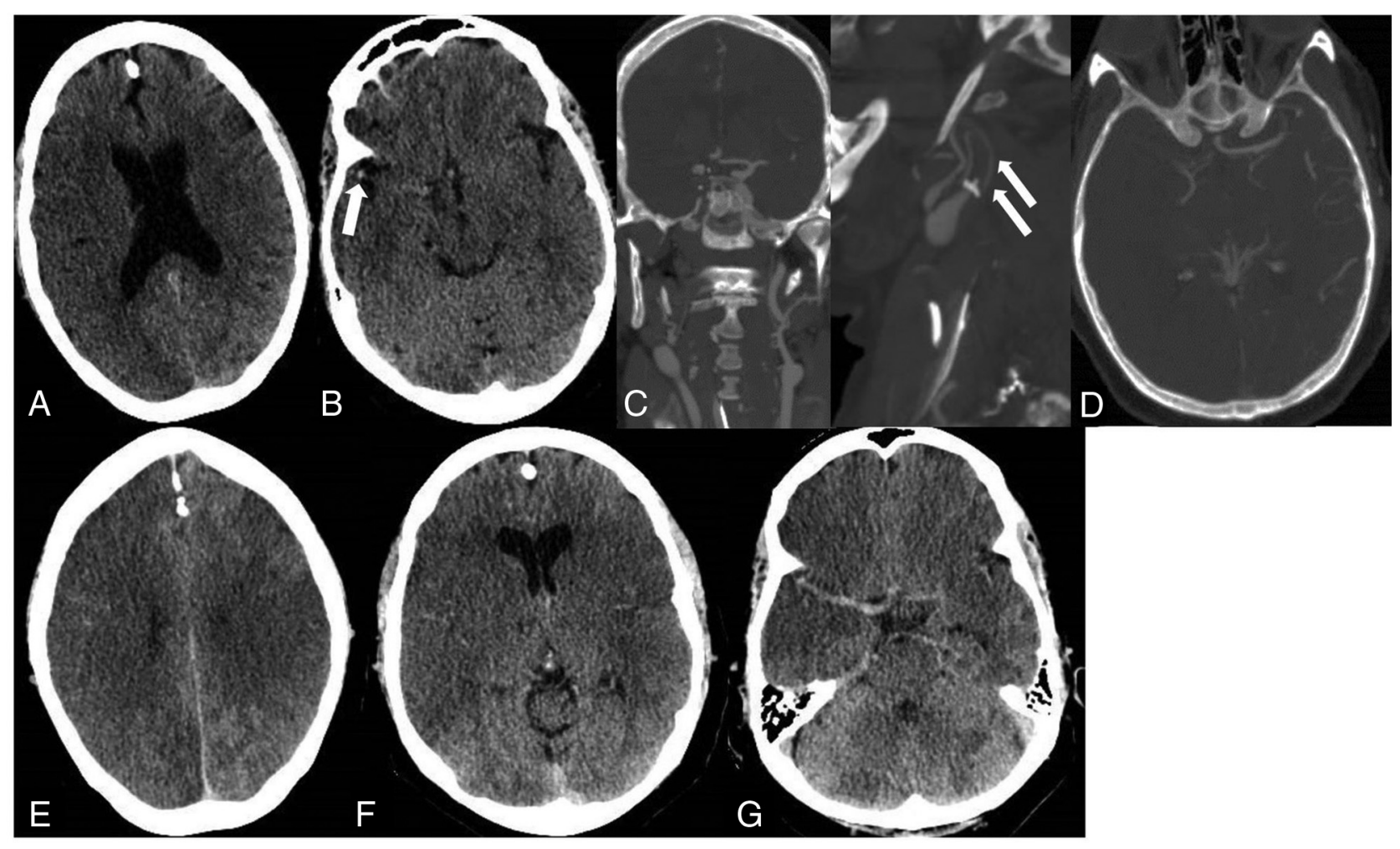

FIG 10. Non-contrast CT demonstrates low attenuation suggestive of an acute right MCA territory infarction (A). There is subtle high attenuation (arrow) in the right Sylvian fissure suggesting thrombus (B). Coronal and sagittal CTA views demonstrate long-segment thrombus resulting in occlusion (arrow) of the right internal carotid artery (C). Head view (D) demonstrates occlusion of the right middle cerebral artery. Follow-up head CT obtained on the same day as CTA reveals a right middle cerebral artery territory acute infarction and possible infarctions in the left parietal lobe and left temporal region $(E$ and $F$ ). Cerebellar infarctions were also present $(G)$.

megakaryocytes, platelets, fibrin, and inflammatory cells, eg, neurophils. ${ }^{14}$ Similarly, postmortem examinations from the SARS outbreak of the early 2000s demonstrated pulmonary thrombi, pulmonary infarcts, and microthrombi in other organs. ${ }^{6,7}$

Patients infected with COVID-19 may have a massive inflammatory reaction, presumably due to rapid accumulation of T-cells and macrophages releasing cytokines into the bloodstream, which aim to destroy the virus, resulting in CSS. ${ }^{4,5}$ Laboratory findings of CSS include decreased T-cells and natural killer cells and an increase in interleukin 6. Hypoxia is a trigger of inflammation and contributes to an intense proinflammatory state..$^{15,16}$ In addition, the COVID-19 virus has an affinity for ACE2 receptors, which are found in the capillary endothelium., ${ }^{2,3}$ Data suggest that engagement of the endothelium by the virus damages it, increasing the permeability of the blood-brain barrier, resulting in encephalopathy, encephalitis, and thrombosis and hemorrhage. ${ }^{17-19}$ Hemorrhages may be related to a coagulopathy due to endothelial dysfunction as sequelae of CSS and/or liver dysfunction and consumption of clotting factors leading to disseminated intravascular coagulation.

Neurologic manifestations are now recognized and, in 1 series, were present in $36.4 \%$ of patients. ${ }^{20,21}$ These findings may be, at least in part, due to the affinity of COVID-19 for the ACE2 receptors, which are relatively common in the respiratory and nervous systems. ${ }^{2,3}$ Occasionally, CNS symptoms precede other manifestations and may be the only indicators of the disease. ${ }^{21}$ Postmortem examinations have shown brain hyperemia and edema as well as neuronal degeneration in patients with COVID-19. ${ }^{22}$

As in other series, most of our patients (8 of 10) had significant comorbidities, more commonly hypertension $(n=6)$, morbid obesity $(n=4)$, diabetes $(n=3)$, and cancer $(n=1)$. Obesity is associated with a risk of stroke, not only due to the propensity for comorbid diabetes, hypertension, and hyperlipemia, all independent risk factors for cardiovascular disease, but also because fatty tissues are inherently proinflammatory and hypofibrinolytic. ${ }^{23}$ Being overweight is also a documented risk for poor outcome in COVID-19 infection, ${ }^{24}$ and its link to an enhanced inflammatory state and inherent prothrombotic state may also explain the high prevalence of cerebral infarctions in these patients. The number of cases herein described is too small to determine whether specific comorbidities lead to specific CNS manifestations. The high number of comorbidities in our patients may explain why only 3 were older than 65 years when advancing age has been a predisposing factor in other series.

We cannot speculate on the frequency of cerebral infarctions in our patients with COVID-19 because the cases shown here were specifically chosen to review these complications. In 1 large series, slightly $>36 \%$ of patients had CNS complications. ${ }^{21}$ In that same series, 88 of 214 patients had severe infections, and they were the ones with CNS complications. Of these patients, 4 had ischemic strokes and 1 a cerebral hemorrhage. Most them also had severe hypertension, which was present in most of our patients. In a small series from France, 13 patients underwent 
brain MR imaging and 3 showed cerebrovascular abnormalities, which, by description, were small compared with the ones in our series, and 8 showed leptomeningeal enhancement, which we saw in only 1 patient. $^{25}$ One of our patients showed diffuse cerebral edema, and it is unclear whether this was the result of anoxia induced by lung disease or a different reason. It is notable that 6 of our patients had symptoms and laboratory values suggesting CSS but their brain imaging findings varied widely, and none corresponded to findings described previously for this syndrome. ${ }^{20}$

Thus, it seems that the neurovascular complications of COVID-19, as illustrated by our cases and those of others, ${ }^{26-30}$ are common and varied, including multifocal infarctions, multiple arterial occlusions, and/or intraluminal thrombi with or without hemorrhages, which may be the result of a combination of diffuse endothelial dysfunction, systemic hypercoagulability, and hypoxic-ischemic damage from cardiopulmonary dysfunction. ${ }^{21}$ Previously, we reported posterior reversible encephalopathy syndrome-like findings in 2 patients, whom we decided not to include in these series. ${ }^{31}$

\section{CONCLUSIONS}

We presented 10 hospitalized patients with COVID-19 who had severe intracranial ischemic complications, and most had comorbidities, including hypertension, morbid obesity, and diabetes. Although probably multifactorial, in many patients, neurovascular ischemic diathesis in the setting of COVID-19 infection may be explained by a combination of cytokine release syndrome resulting in severe inflammation, leading to endothelial dysfunction; a hypercoagulable state; and cardiopulmonary dysfunction with hypoxia and hemodynamic insufficiency accentuated by direct viral-mediated breakdown of the blood-brain barrier. Although our study is a small observational series, it demonstrates the wide range of ischemic brain lesions in patients with COVID-19, and these should be kept in mind when imaging such patients.

\section{REFERENCES}

1. Geneva: World Health Organization. Coronavirus disease (COVID19) pandemic. https://www.who.int/emergencies/diseases/novelcoronavirus-2019. Accessed April 30, 2020

2. Hamming I, Timens W, Bulthuis MLC, et al. Tissue distribution of ACE2 protein, the functional receptor for SARS coronavirus: a first step in understanding SARS pathogenesis. J Pathol 2004;203: 631-37 CrossRef Medline

3. Li WH, Moore MJ, Vasilieva NY, et al. Angiotensin-converting enzyme $\mathbf{2}$ is a functional receptor for the SARS coronavirus. Nature 2003;426:450-54 CrossRef Medline

4. Mehta P, McAuley DF, Brown M, et al; HLH Across Speciality Collaboration, UK. COVID-19: consider cytokine storm syndromes and immunosuppression. Lancet 2020;395:1033-34 CrossRef Medline

5. Wang W, He J, Lie $\mathrm{P}$, et al. The definition and risks of cytokine release syndrome-like in 11 COVID-19-infected pneumonia critically ill patients: disease characteristics and retrospective analysis. medRxiv February 27, 2020. https://doi.org/10.1101/2020.02.26. 20026989. Accessed April 30, 2020

6. Ding Y, He L, Zhang Q, et al. Organ distribution of severe acute respiratory syndrome (SARS) associated coronavirus (SARS-CoV) in
SARS patients: implications for pathogenesis and virus transmission pathways. J Pathol 2004;203:622-30 CrossRef Medline

7. Ding $\mathrm{Y}$, Wang $\mathrm{H}$, Shen $\mathrm{H}$, et al. The clinical pathology of severe acute respiratory syndrome (SARS): a report from J Pathol 2003; 200:282-89 CrossRef Medline

8. Klok FA, Kruip M, van der Meer NJ, et al. Incidence of thrombotic complications in critically ill ICU patients with COVID-19. Thromb Res 2020;191:145-7 CrossRef Medline

9. Xie J, Tong Z, Guan X, et al. Clinical characteristics of patients who died of coronavirus disease 2019 in China. JAMA Netw Open 2020; 3:e205619 CrossRef Medline

10. Ruan Q, Yang K, Wang W, et al. Clinical predictors of mortality due to COVID-19 based on an analysis of data of 150 patients from Wuhan, China. Intensive Care Med 2020;46:846-48 CrossRef Medline

11. Jin Y, Cai L, Cheng Z, et al; for the Zhongnan Hospital of Wuhan University Novel Coronavirus Management and Research Team, Evidence-Based Medicine Chapter of China International Exchange and Promotive Association for Medical and Health Care (CPAM). A rapid advice guideline for the diagnosis and treatment of 2019 novel coronavirus (2019-nCoV) infected pneumonia (standard version). Mil Med Res 2020;7:4 CrossRef Medline

12. Wadman M. How does coronavirus kill? Clinicians trace a ferocious rampage through the body, from brain to toes. Science 2020 April 17 CrossRef

13. Poor HD, Ventetuolo CE, Tolbert T, et al. COVID-19 critical illness pathophysiology driven by diffuse pulmonary thrombi and pulmonary endothelial dysfunction responsive to thrombolysis. Clinical and Translational Medicine May 13, 2020. https://onlinelibrary.wiley. com/doi/abs/10.1002/ctm2.44. Accessed May 15, 2020

14. Fox SE, Akmatbekov A, Harbert JL, et al. Pulmonary and cardiac pathology in Covid-19: the first autopsy series from New Orleans. Research Gate April 2020. https://www.researchgate.net/publication/ 340563942_Pulmonary_and_Cardiac_Pathology_in_Covid-19_ The_First_Autopsy_Series_from_New_Orleans. Accessed April 30, 2020

15. Eltzschig HK, Carmeliet P. Hypoxia and inflammation. $N$ Engl J Med 2011;364:656-65 CrossRef Medline

16. Bartels K, Grenz A, Eltzschig HK. Hypoxia and inflammation are two sides of the same coin. Proc Natl Acad Sci U S A 2013;110: 18351-52 CrossRef Medline

17. Baig AM, Khaleeq A, Ali U, et al. Evidence of the COVID-19 virus targeting the CNS: tissue distribution, host-virus interaction, and proposed neurotropic mechanisms. ACS Chem Neurosci 2020;11: 995-98 CrossRef Medline

18. Netland J, Meyerholz DK, Moore S, et al. Severe acute respiratory syndrome coronavirus infection causes neuronal death in the absence of encephalitis in mice transgenic for human ACE2.J Virol 2008;82:7264-75 CrossRef Medline

19. Li YC, Bai WZ, Hashikawa T. The neuroinvasive potential of SARS-CoV2 may play a role in the respiratory failure of COVID-19. J Med Virol 2020 Feb 27. [Epub ahead of print] CrossRef Medline

20. Filatov A, Sharma P, Hindi F, et al. Neurological complications of coronavirus (COVID-19): encephalopathy. Cureus 2020;12:e7352 CrossRef Medline

21. Mao L, Jin H, Wang M, et al. Neurologic manifestations of hospitalized patients with coronavirus disease 2019 in Wuhan, China. JAMA Neurol 2020 Apr 10. [Epub ahead of print] CrossRef Medline

22. National Health Commission of the People's Republic of China. Diagnosis and treatment of the novel coronavirus pneumonia. 2020 http://www.nhc.gov.cn/. Accessed April 15, 2020

23. Blokhin IO, Lentz SR. Mechanisms of thrombosis in obesity. Curr Opin Hematol 2013;20:437-44 CrossRef Medline

24. Petrilli CM, Jones SA, Yang J, et al. Factors associated with hospital admission and critical illness among 5279 people with coronavirus 
disease 2019 in New York City: prospective cohort study. BMJ 2020;369:m1966 CrossRef Medline

25. Helms J, Kremer S, Merdji H, et al. Neurologic features in severe SARS-CoV-2 infection. N Engl J Med 2020 Apr 15. [Epub ahead of print] CrossRef Medline

26. Avula A, Nalleballe K, Narula N, et al. COVID-19 presenting as stroke. Brain Behav Immun 2020 Apr 28. [Epub ahead of print] CrossRef Medline

27. Beyrouti R, Adams ME, Benjamin L, et al. Characteristics of ischaemic stroke associated with COVID-19. J Neurol Neurosurg Psychiatry 2020 Apr 30. [Epub ahead of print] CrossRef Medline

28. Aggarwal G, Lippi G, Henry M. B. Cerebrovascular disease is associated with an increased disease severity in patients with coronavirus disease 2019 (COVID-19): a pooled analysis of published literature. Int J Stroke 2020 Apr 20. [Epub ahead of print] CrossRef Medline

29. Oxley TJ, Mocco J, Majidi S, et al. Large-vessel stroke as a presenting feature of Covid-19 in the young. N Engl J Med 2020;382:e60 CrossRef Medline

30. Radmanesh A, Raz E, Zan E, et al. Brain imaging utilization and findings in COVID-19: A single academic center experience in the epicenter of disease in the United States. AJNR Am J Neuroradiol 2020 May 28. [Epub ahead of print] CrossRef Medline

31. Franceschi AM, Ahmed O, Giliberto L, et al. Hemorrhagic Posterior Reversible Encephalopathy Syndrome as a Manifestation of COVID-19 Infection. AJNR Am J Neuroradiol 2020 May 21. [Epub ahead of print] CrossRef Medline 